\title{
Darrell G. Schlom and James F. Scott Named 2008 MRS Medalists
}

The Materials Research Society has selected Darrell G. Schlom of Cornell University (formerly at the Pennsylvania State University) and James F. Scott of Cambridge University, UK, for a joint 2008 MRS Medal for "fundamental contributions to the materials science of oxides underlying current and future electronic devices." The Medalists will be recognized during the awards ceremony at the 2008 MRS Fall Meeting in Boston on Wednesday, Dec. 3, at 6:00 p.m. in the Grand Ballroom at the Sheraton Boston Hotel. On Thursday, Dec. 4, they will give their award talks in the Grand Ballroom. Scott will speak at 12:05 p.m. on "Some New Properties of Ferroelectrics-THz Emission, Nanodomain Switching, Finite-Size Effects, and Ferroelectrically-InducedFerromagnetism,"and Schlom will deliver his talk at 12:45 p.m. on "Oxides Beyond $\mathrm{SiO}_{2}$ and the High-K Materials Revolution."

Darrell G. Schlom has many accomplishments in the broad area of complex oxide electronics, but this award is specifically focused on his contributions to the field of next-generation of oxides for gate dielectrics. The pioneering work of Schlom's group at Penn State, over a decade ago, has led to a major materials revolution in computers: after more than 40 years, the $\mathrm{SiO}_{2}$ gate dielectric of $\mathrm{Si}-$ based transistors is being replaced by a material with a much higher dielectric constant $(K), \mathrm{HfO}_{2}$. Schlom's group comprehensively analyzed the thermodynamic stability of oxides in contact with silicon and was the first to suggest that the $\mathrm{HfO}_{2} / \mathrm{Si}$ interface was stable. A number of computer companies have either implemented, or announced plans, to replace the $\mathrm{SiO}_{2}$ gate insulator of conventional transistors by an $\mathrm{HfO}_{2}$ dielectric, a development which saves power at the same time as it boosts speed.

After obtaining a PhD degree in the Materials Science and Engineering (MSE) department at Stanford University, in 1990, Schlom accepted a postdoctoral research fellowship at the IBM Research Laboratory in Zürich, Switzerland, after which he joined the MSE faculty at the Pennsylvania State University in 1992. He was appointed associate professor in the same department in 1998, professor in 2002, and distinguished professor in 2007.

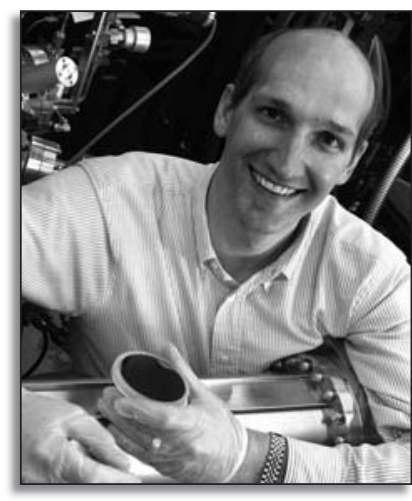

Darrell G. Schlom

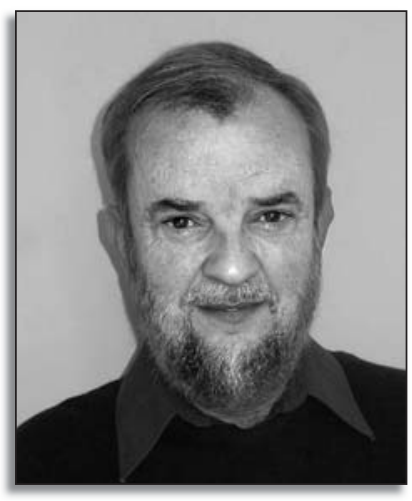

James F. Scott
He joined the faculty at Cornell University in 2008. Schlom was an Alexander von Humboldt Research Fellow at the University of Augsburg, Germany, from 1999 to 2000. He has co-authored over 300 publications, with an $h$-index of 43 and more than 7000 citations. He also has had seven patents granted, with three pending. His many awards include the IBM Invention Achievement Award (1991), the NSF Young Investigator Award and the ONR Young Investigator Award (1993), the Semiconductor Research Corporation Inventor Recognition Award (2004), and the Penn State Faculty Scholarship Medal in Engineering (2006). Schlom was elected fellow of the American Physical Society in 2003. He was elected to the MRS Board of Directors (2005-2007) and appointed to the Defense Science Study Group (2004-2005).

James F. Scott has made several landmark contributions in areas impacting a broad range of advanced technologies. His primary focus is at the interface between device physics and enabling technologies of complex functional materials. $\mathrm{He}$ is a leader in the field of ferroelectric and, more generally, ferroic materials, in particular in the study of ferroelectric thin films. Scott's ability to take advantage of the most prominent property of ferroelectrics, their two stable states of opposite polarization, has led the way to their application to ultrafast electronic memories. For example, Scott's work on strontium bismuth tantalate (SBT), which he initiated in the early 1990s, is now being applied in advanced product development, for applications including 60-nm capacitors for the $45-\mathrm{nm}$ node in semiconductors by
Matsushita/Symetrix, and a mixed-signal chip with analog, digital, and microprocessors, which is used in the Japanese Rail-Road "smart card" fare-card. As a scientist, he has led cutting-edge research in ferroelectric thin films, their structure, and properties, in particular, the way thin-film processing influences their performance.

Scott obtained a $\mathrm{PhD}$ degree in physics at the Ohio State University in 1966, after which he joined the Quantum Electronics Research Department, Bell Telephone Laboratories, NJ. In 1972, Scott was appointed Professor of Physics at the University of Colorado, where he remained until 1992. He was appointed Dean of Applied Science at the Royal Melbourne Institute of Technology, Australia (19921995), and Dean of Science and Professor of Physics at the University of New South Wales in Sydney, Australia (1995-1999). Since 1999, Scott has been Professor of Ferroics, Cambridge University. Scott was awarded the Humboldt Prize (Halle/ Jülich) in 1997 and the Monkasho Prize (Nara) in 2001, and he was elected a Fellow of the Royal Society (FRS) in 2008. Scott was a director on the Board of Technical Advisors at Ramtron Corp. (1984-1986), and chair of the Board of Directors at Symetrix Corp. (1986-1990). He has authored or co-authored more than 400 journal articles, with an $h$-factor of 50 and more than 10,000 citations. He has edited five books and is author of the book Ferroelectric Memories (Springer, 2000), which has been translated into Japanese by Springer-Japan (2003), and into Chinese by Tsinghua Press (2004). 